\title{
Open charm hadron production via hadronic decays at STAR
}

\author{
David Tlustý for the STAR collaboration \\ ${ }^{a}$ Nuclear Physics Institute, Academy of Sciences Czech Republic, Na Truhlářce 39/64, 18086 Praha 8, Czech Republic \\ ${ }^{b}$ Czech Technical University in Prague, Faculty of Nuclear Sciences and Physical Engineering, Břehová 7, 11519, Prague 1, Czech Republic
}

\begin{abstract}
Heavy quarks are a unique probe to study the medium produced in ultra-relativistic heavy ion collisions. The dominant process of charm quark production at RHIC is believed to be initial gluon fusion which can be calculated in the perturbative QCD. The upper limit of FONLL calculation seems to be in good agreement with charm cross section measurements at mid-rapidity in $p+p$ collisions at $\sqrt{s_{N N}}=200 \mathrm{GeV}$ provided by STAR. The same measurement in Au+Au collisions at equal energy reveals the numberof-binary-collisions scaling of charm cross section indicating that charm production is dominated by initial hard scatterings. In this article, we report the measurements of $D^{0}, D^{*}$ in $p+p$ at $0.6 \mathrm{GeV} / c<p_{T}<6 \mathrm{GeV} / c$ and $D^{0}$ in $\mathrm{Au}+\mathrm{Au}$ collisions at 0.2 $\mathrm{GeV} / c<p_{T}<5 \mathrm{GeV} / c$ via hadronic decays $D^{0} \rightarrow K^{-} \pi^{+}, D^{*+} \rightarrow D^{0} \pi^{+} \rightarrow K^{-} \pi^{+} \pi^{+}$at mid-rapidity $|y|<1$.
\end{abstract}

Keywords: STAR, QGP, Heavy Flavor, Open Charm

\section{Introduction}

The charm quark production is dominated by initial gluon fusion at initial hard partonic collisions and well described by perturbative QCD (pQCD) because of large charm quark mass $\left(\sim 1.5 \mathrm{GeV} / \mathrm{c}^{2}\right)[1]$. The mass is almost exclusively generated through its coupling to the Higgs field in the electroweak sector, while masses of (u, d, s) quarks are dominated by spontaneous breaking of chiral symmetry (CS) in QCD [2]. This means that charm quarks remain heavy even if CS is restored, as it likely is in a QGP. Since charm is produced mainly in initial hard scatterings, one expects that charm production total cross section $\sigma_{c \bar{c}}^{N N}$ should scale as a function of number-of-binary-collisions $N_{\text {bin. }}$. In addition, if charm quarks flow elliptically, there must have been enough interactions to easily thermalize light quarks. Hence, charm quarks are an ideal probe to study early dynamics in high-energy nuclear collisions.

\section{Measurement}

Charm cross section at mid-rapidity is calculated from open charm hadrons yields. These yields are obtained from the invariant mass reconstruction of open charm mesons through hadronic decays: $D^{0}\left(\overline{D^{0}}\right) \rightarrow K^{\mp} \pi^{ \pm}(\mathrm{BR}=3.89 \%)$ and $D^{* \pm} \rightarrow D^{0}\left(\overline{D^{0}}\right) \pi^{ \pm}(\mathrm{BR}=67.7 \%) \rightarrow K^{-} \pi^{+} \pi^{ \pm}$. The identification of daughter particles was done in the STAR experiment (see Fig. 1) at mid-rapidity $|y|<1$ at $\sqrt{s_{N N}}=200 \mathrm{GeV}$.

The analysis presented herein was done using two datasets; the first one collected in 2009 with minimum bias trigger defined as a coincidence in Vertex Position Detectors East and West (105 million events total) and the second one collected in 2010 with minimum bias trigger defined as a signal in Zero Degree Calorimeter (280 million events total).

Sub-detectors used in this analysis were: 


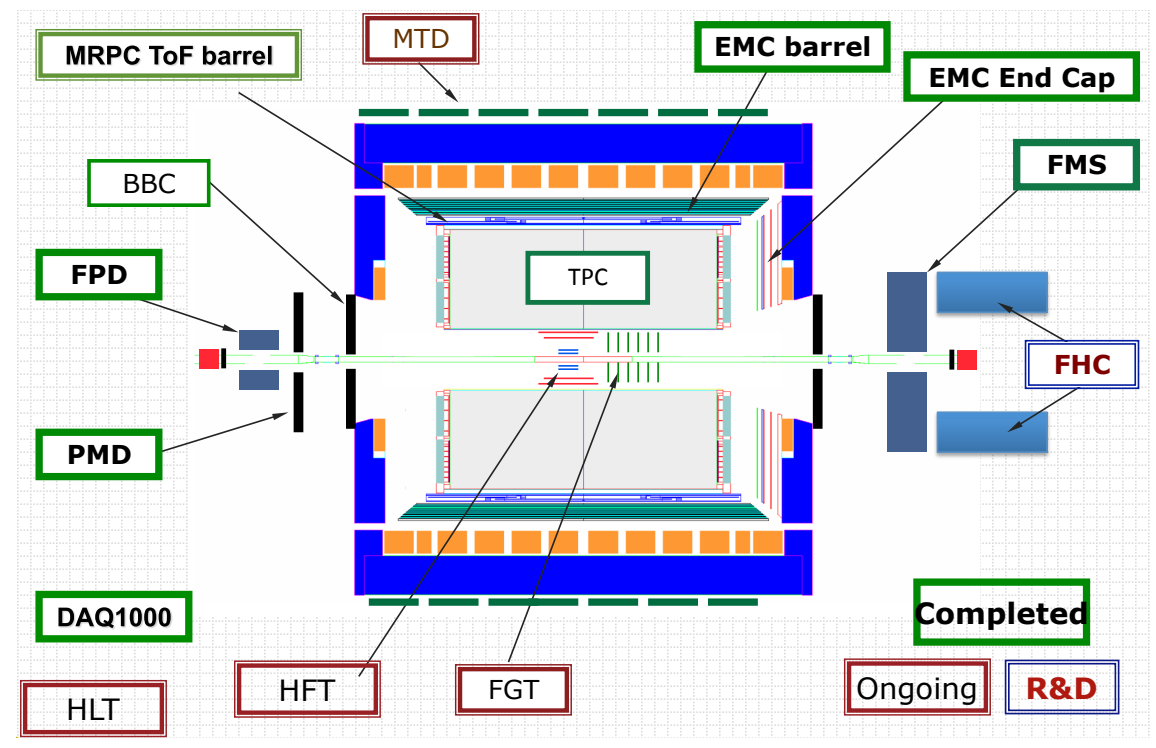

Figure 1. The STAR detector. TPC (Time Projection Chamber) is main detector for tracking and PID (providing $\mathrm{d} E / \mathrm{d} x, \vec{p}$ ), TOF (Time Of Flight) for PID improvement, and pile-up tracks removal and BEMC (Barrel Electromagnetic Calorimeter) in this analysis for pile-up tracks removal if TOF is not available.

- Time Projection Chamber (TPC) providing 3D image of a particle track and ionization energy loss $(\mathrm{d} E / \mathrm{d} x)$ and covering a large acceptance with full $2 \pi$ azimuthal angle at $|\eta|<1$. With the help of uniform magnetic field one can calculate momentum vector of a track as a function of track helix radius and magnetic induction.

- Time-Of-Flight (TOF) detector providing time of the particle's flight from primary vertex to a channel pad and covering $72 \%$ in 2009 and $100 \%$ in 2010 of the whole barrel. When combined with momentum measurements from the TPC, this allows to separate pions from kaons up to $1.6 \mathrm{GeV} / \mathrm{c}$ [4].

STAR doesn't have any subsystem being able to reconstruct secondary vertex of $D^{0}$ or $D^{*}$ decay; one must calculate invariant mass of all $K \pi$ pair coming from vicinity of primary vertex. This results in a large combinatorial background which was reconstructed via mixed-event method ( $\mathrm{Au}+\mathrm{Au}$ dataset), same-charge-sign, and kaon momentumrotation ( $\mathrm{p}+\mathrm{p}$ dataset) and subtracted from invariant mass spectra of daughter particle pairs [5]. To reconstruct $D^{*}$, one may exploit the softness of $D^{*} \rightarrow D^{0} \pi$ decay; combine low momentum pions with $D^{0}$ candidates, i.e. pairs with $1.82<M(K \pi)<1.9 \mathrm{GeV} / \mathrm{c}$, and plot difference $M(K \pi \pi)-M(K \pi)$. Such value occupies the beginning of the phase space; hence it does not suffer from large combinatorial background making $D^{*}$ direct observation possible. The combinatorial background was reconstructed by side-band (picking $D^{0}$ candidates outside the $D^{0}$ mass region) and wrong-sign (picking soft pion with opposite charge) methods. The difference between these methods is the dominant source of systematic uncertainties for both $D^{0}$ and $D^{*}$ analyses.

Open charm hadrons yields $Y_{D^{0}, D^{*}}$ were calculated as Gaussian function areas from the invariant mass peaks fits shown in Figure 2 Raw counts were corrected with the reconstruction efficiency in used sub-detectors to obtain the correct $Y_{D^{0}, D^{*}}$.

\section{RESULTS}

Yields were calculated in $6 p_{T}$ bins ( 2 for $D^{0}, 4$ for $D^{*}$ ) in $\mathrm{p}+\mathrm{p}$ dataset and $5 p_{T}$ bins (all for $D^{0}$ ) in $\mathrm{Au}+\mathrm{Au}$ dataset. Then the invariant charm cross section $\mathrm{d} \sigma_{\mathrm{p}+\mathrm{p}}^{c \bar{c}} /\left(2 \pi p_{T} \mathrm{~d} p_{T} \mathrm{~d} y\right)$ was calculated by formula

$$
\frac{\mathrm{d} \sigma^{c \bar{c}}}{2 \pi p_{T} \mathrm{~d} p_{T} \mathrm{~d} y}=\frac{Y \sigma^{\mathrm{NSD}}}{2 \pi p_{T} \Delta p_{T} \Delta y \mathrm{BR}} \frac{\epsilon_{\mathrm{T}}}{N f_{\text {frag. }}}
$$



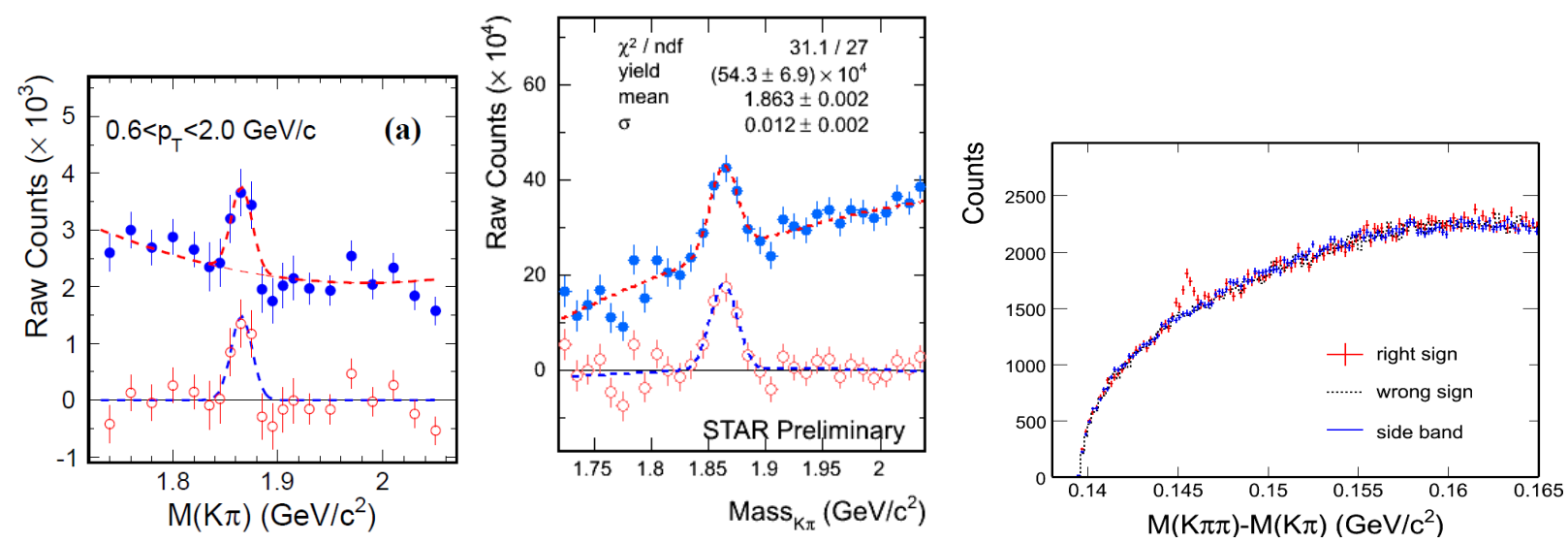

Figure 2. Left panel: $D^{0}$ signal in $\mathrm{p}+\mathrm{p} 200 \mathrm{GeV}$ collisions after same-sign background subtraction. Middle panel: $D^{0}$ signal in Au+Au $200 \mathrm{GeV}$ collisions after mixed-event background subtraction Right panel: $D^{*}$ signal in $\mathrm{p}+\mathrm{p} 200 \mathrm{GeV}$ collisions. Combinatorial background is reproduced by the distributions from the wrong-sign (black dotted) and side-band (blue solid) methods.

in each $p_{T}$ bin $\left(\sigma^{\mathrm{NSD}}\right.$ is non-single diffractive $\mathrm{p}+\mathrm{p}$ inelastic cross section, $f_{\text {frag. }}$ is the ratio of charm quarks hadronized to open charm mesons and $\epsilon_{\mathrm{T}}$ is the trigger bias correction). The charm cross section at mid rapidity $\mathrm{d} \sigma^{c \bar{c}} / \mathrm{d} y$ was obtained from power-law function fit to $\mathrm{d} \sigma^{c \bar{c}} /\left(2 \pi p_{T} \mathrm{~d} p_{T} \mathrm{~d} y\right)$ points (see Fig. 3 ) as $170 \pm 45$ (stat. $)_{-51}^{+37}$ (sys.) $\mu \mathrm{b}$. In $\mathrm{Au}+\mathrm{Au}$ collisions we calculated invariant yield $\mathrm{d}^{2} N /\left(N_{\mathrm{ev}} p_{T} \mathrm{~d} p_{T} \mathrm{~d} y\right)$.
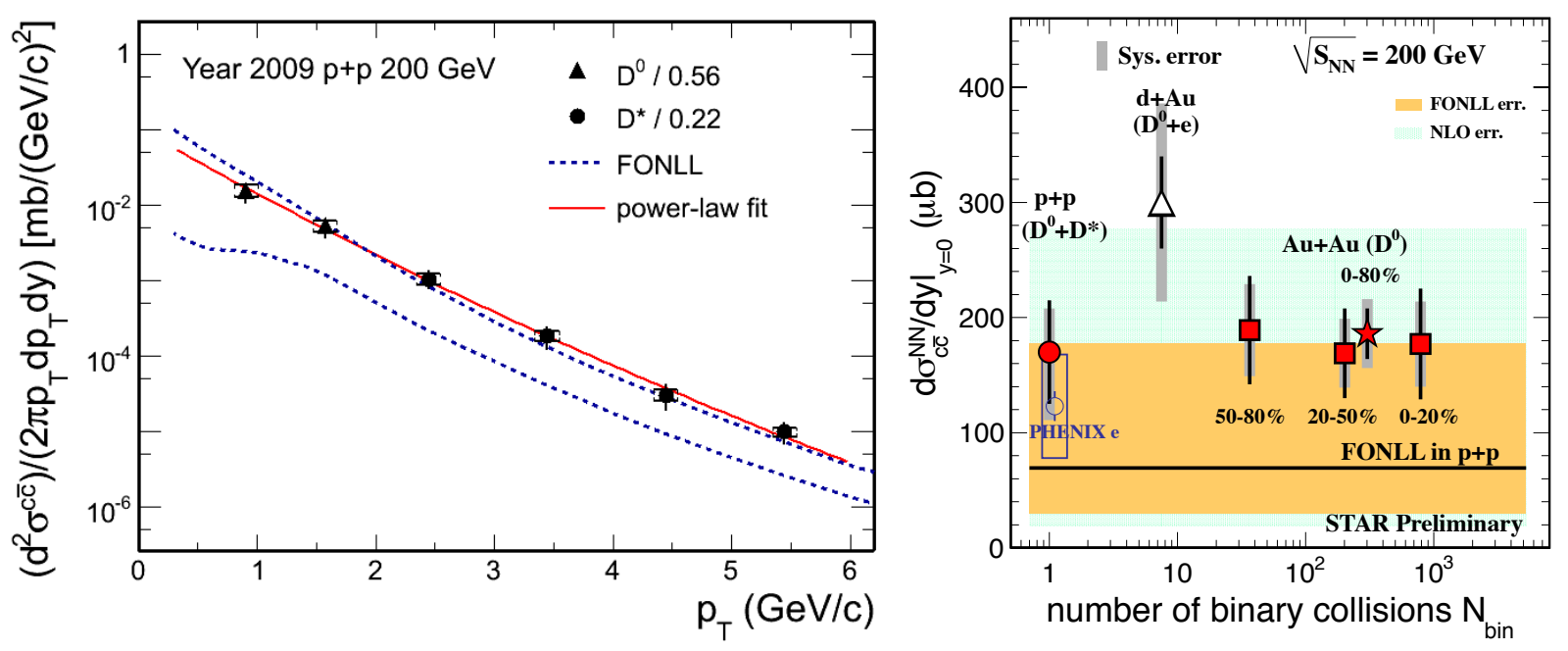

Figure 3. Left Panel: $c \bar{c}$ pair production cross section (symbols) as a function of $p_{T}$ in $200 \mathrm{GeV}$ p+p collisions. Right Panel: The charm production cross section per nucleon-nucleon collision at mid-rapidity as a function of $N_{\text {bin }}$.

The $\mathrm{d} \sigma^{c \bar{c}} / \mathrm{d} y$ at mid-rapidity in Au+Au collisions was extracted, from a power-law fit as $186 \pm 22$ (stat.) \pm 30 (sys.) \pm 18 (norm.) $\mu \mathrm{b}$ assuming that the $f_{\text {frag. }}$ does not change from $\mathrm{p}+\mathrm{p}$ to $\mathrm{Au}+\mathrm{Au}$ collisions. The charm cross section for three centrality bins, $0-20 \%, 20-50 \%$ and $50-80 \%$, is obtained according to the integrated yields. The charm production cross section per nucleon-nucleon collision at mid-rapidity as a function of $N_{\text {bin }}$ is shown in the right panel of Fig. 3. Within errors, the results are in agreement and follow the number of binary collisions scaling, which indicates that charm quark is produced via initial hard scatterings at early stage of the collisions at RHIC. The FONLL (orange band) and NLO [6] (light-blue band) uncertainties are also shown here for comparison.

The $R_{\text {AA }}^{D^{0}}$ depicted in Figure 4 was obtained via dividing $D^{0}$ yields in $\mathrm{Au}+\mathrm{Au} 0-80 \%$ minbias collisions by the power-law fit to $\mathrm{p}+\mathrm{p}$ yields scaled by $N_{\text {bin }}$, The uncertainty of the $\mathrm{p}+\mathrm{p}$ power-law shape is taken into account as 


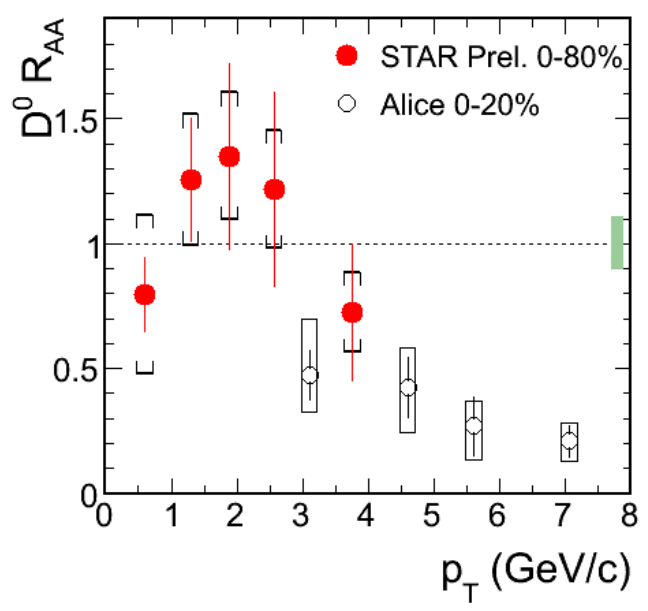

Figure 4. $D^{0}$ nuclear modification factor $R_{\mathrm{AA}}$ as a function of p. ALICE data taken from 7

systematic error. No suppression is observed at $p_{T}<3 \mathrm{GeV} / \mathrm{c}$.

\section{CONCLUSIONS}

Open charm hadrons $\left(D^{0}, D^{*+}\right)$ are measured in $\mathrm{p}+\mathrm{p}$ and $\mathrm{Au}+\mathrm{Au}$ collisions at $\sqrt{s_{\mathrm{NN}}}=200 \mathrm{GeV}$ at STAR. Charm cross sections per nucleon-nucleon collision at mid-rapidity follow the number of binary collisions scaling. The charm pair production cross sections per nucleon-nucleon collision at mid rapidity are measured to be $\mathrm{d} \sigma^{c \bar{c}} /\left(2 \pi p_{T} \mathrm{~d} p_{T} \mathrm{~d} y\right)=$ $170 \pm 45$ (stat.) ${ }_{-51}^{+37}$ (sys.) $\mu \mathrm{b}$ in $\mathrm{p}+\mathrm{p}$ and $186 \pm 22$ (stat.) \pm 30 (sys.) \pm 18 (norm.) $\mu \mathrm{b}$ in $\mathrm{Au}+\mathrm{Au}$ minimum bias collisions. In the near future the STAR Heavy Flavor Tracker [8] will provide the necessary resolution to reconstruct secondary vertices of charm mesons, which will increase the precision of charm measurements.

\section{Acknowledments}

This work was supported by grant INGO LA09013 of the Ministry of Education, Youth and Sports of the Czech Republic, and by the Grant Agency of the Czech Technical University in Prague, grant No. SGS10/292/OHK4/3T/14.

\section{References}

[1] M. Cacciari, P. Nason and R. Vogt, Phys. Rev. Lett. 95, 122001 (2005).

[2] X. Zhu, et al., PLB 647, 366(2007)

[3] B. I. Abelev et al., Phys. Rev. Lett. 98, 192301 (2007).

[4] M. Shao et al., Nucl. Instrum. Methods A 499, 624 (2003).

[5] J. Adams et al., Phys. Rev. Lett. 94, 062301(2005).

[6] R. Vogt, Eur.Phys.J.ST 155 213, (2008).

[7] A. Grelli et al., SQM 2011 Conference Proceedings, arXiv:1201.2262.

[8] Z. Xu et al., J. Phys. G 32, S571(2006). 